\title{
Competitive Intelligence Monitoring in the Risk Prevention of SMEs
}

\author{
Xianjin ZHA, Minghong CHEN \\ Center for Studies of Information Resources, Wuhan University, Wuhan, China. \\ Email: xianjinzha@163.com
}

Received June $17^{\text {th }}$, 2009; revised July 24 ${ }^{\text {th }}$ 2009; accepted August $2^{\text {nd }}, 2009$.

\begin{abstract}
Competitive Intelligence plays an increasingly important role in the risk prevention of SMEs, however, there is an obvious phenomenon-the absence of competitive intelligence in the field of Chinese SMEs. In this paper, the author firstly pointed out how many kinds of risks the SMEs were facing, and then analyzed the demand for competitive intelligence in the risk prevention of SMEs. Based on that, the model of competitive intelligence monitoring in the risk prevention of SMEs were put forward at last.
\end{abstract}

Keywords: small and medium-sized enterprises (SMEs), risk prevention, competitive intelligence

\section{Introduction}

With the change of economic environment and fierce competition between large enterprises, many small and medium enterprises (SMEs) survive difficultly, for they are facing increasingly diversified and complicated risks in the activities of production, operation, management and decision-making. Thus people begin to explore how SMEs could convert from enduring consequences of risks passively to using risks positively to create value.

Competitive intelligence, which is developed in the course of competitive activities, involving collecting and extracting the information about competitors, competitive environments and competitive strategies, aims to win and maintain competitive advantages of enterprises [1]. Its successful application helps SMEs to win competitive advantages by identifying the potential threats and opportunities in the market as soon as possible and by reducing competitors' response time, equal to increasing their own response time. To be specific, competitive intelligence not only facilitates risk management by predicting, identifying, avoiding, transferring, spreading and controlling risks well, but also helps SMEs to enhance the capabilities of risk awareness and risk prevention. In this paper, competitive intelligence is regarded as the breakthrough point to study how SMEs prevent risks in the productive and commercial activities.

\section{SMEs and their Risks}

Generally speaking, compared with large enterprises in the same industry, SMEs are smaller economic unitsfewer employees, less assets and smaller scale. And the modified definitions of SMEs are not the same in the different countries, development stages and industries, however, the quality and the quantity are still basic parameters for the definition of SMEs [2].

In fact, SMEs often play a role of vulnerable groups in the fierce market competition in contrast with these large enterprises. According to the report of Chinese Academy of Social Sciences on the role of SMEs in the recovery of financial crisis, $40 \%$ of SMEs have been collapsed, $40 \%$ of SMEs are struggling against the financial crisis, and only $20 \%$ of SEMs are immune to the current financial crisis [3]. However, SMEs, having a large number, flexible forms and wide distribution, have been becoming a major strength in the economic and social development process. It means that SMEs are playing an irreplaceable role in promoting economic diversification and in expanding urban and rural employment. Just for this reason, the governments of many countries try to take some laws and regulations to improve the business environment of the SMEs and further to promote the healthy development of SMEs primarily by taking measures in the aspects of financial support, business support, technical innovation, market development and social services, for example, the law "SME Promotion Law of People's Republic of China”. 
Nevertheless, SMEs still face great difficulties. In general, enterprise risks refer either to the extent of losses and the possibility of uncertainties and errors caused by nature, politics, economics, cultures, etc. or to the possibility of the deviation of the reality from the expected or established objectives. Accordingly, enterprise risks consisting in various fields of production, operation, management and decision-making, could be classified into stock risk, inventory risk, market risk, investment risk, technical risk and so on. Furthermore, according to different standards, all the above-mentioned risks are assorted into the various categories: external risk and internal risk, pure risk and speculative risk, fundamental risk and personal risk, realistic risk and potential risk.

However, these risks can be further broken down. For example, external risks can be divided into natural risk, policy risk, legal risk, market risk, industrial risk, competitive risk, the risk of technological innovation, etc.; while internal risks can be divided into strategic risk, investment risk, operational risk, financial risk, technical risk, the risk of human resources, etc. With the establishment of modern enterprise system, SMEs have become independent operators, who regard property rights as a link to the self-risk in the market economy environment. At the same time, the increasingly fierce competition makes risks so unpredictable that a large number of SMEs always face a variety of realistic and potential risks, such as prices decline, the global economic slowdown, and higher interest rates and so on. If SMEs could not take some effective measures to prevent those risks timely, they may find it difficult to survive and develop. And yet most risks, which have more or less something with emergencies, are sudden and ferocious. Therefore, it is impossible for SEMs to make effective countermeasures to handle emergencies well, on condition that they should not identify and prevent those risks in advance.

\section{Demand for Competitive Intelligence in the Risk Prevention of SMEs}

It is obvious that risk prevention is an objective demand for the long-lasting survival and development of SMEs, owing to the uncertainty of internal factors and the complexity of the external environment. In fact, the risk is a kind of uncertainty resulted by asymmetric information and incomplete information. And in most cases, the so-called uncertainty could be predicted and measured through collecting and analyzing information, both of which are just the core work of competitive intelligence.

As a product of market competition and social informationization, competitive intelligence does not only refer to the collection and statistics of data, nor to the answer to specific questions, but to all the continuous and systematical activities of collection and analysis of the full information that may be related to competitiveness. And the focus of competitive intelligence activities directly aims at the competitive environment and competitors. First of all, people carry out market research in a wide range in a multi-directional and multi-level way so as to collect useful information, and after comprehensive analysis and systematic processing, professionals would transform the occupied information into intelligence, which could offer decision-making support to SMEs in several aspects of R\&D, investment strategy and longterm business strategy.

The practice shows that competitive intelligence is so useful to help SMEs improve the ability of risk awareness and risk prevention that they subsequently can be easy to find a shortcut of dealing with risks and further to minimize the losses even if they stand on the edge of a precipice. As a rule, all the evergreen enterprises often attach great importance to competitive intelligence. They have a keen scent for the "clues" of changes in the competitive environment. When the potential threat and risk turned into realities, they could grasp the changing context accurately and respond quickly. As a result, these enterprises would reduce losses, seek potential business opportunities and increase revenue with the help of competitive intelligence.

In this sense, competitive intelligence determines the survival of enterprises. Making full use the favorable characteristics of competitive intelligence, namely, "responding timely" and "avoiding surprise", SMEs could minimize the losses of the crisis at the minimum cost by preventing the risk timely and taking prompt measures to deal with risks.

\section{The Model and Contents of Competitive Intelligence Monitoring in Risk Prevention of SMEs}

Usually, managers are confronted with different kinds of risks (macro-economic, policy, competitive and resource) and some are in fact unique [4]. Managing risk is a complex process that draws upon various levels of management expertise and knowledge, and needs to be placed in the context of managing change. Jones et al. have addressed the issue of readiness for market changes by linking a human relations culture orientation with the usage of a new computing system [5]. This focuses attention on:

- How senior managers are able to devise a strategic intelligence policy that incorporates the use of technology. 
- How competitive intelligence officers can undertake research into technology life cycles.

- How marketing intelligence officers interpret changes in government regulations.

- How global product development teams devise and implement product launches.

- How competitive intelligence officers develop competitor mapping devices.

Obviously, competitive intelligence is advantageous for SMEs to put in place an organizational resilience value system that is created for and supports risk prevention of SMEs, by adopting a proactive approach to risk assessment [6]. What is important to note is that the resilience value system, which is underpinned by the SME's strategic goal, results from the vision and leadership of managers who are committed to establishing an intelligence focus, which has a security dimension of risks. In this paper, it is put out that the model of competitive intelligence monitoring in risk prevention of SMEs and it is shown as Figure 1.

This model shows that competitive intelligence is composed of three components that are competitors, the competitive environment and competitive strategy. And in this model, the whole process of risk prevention, including forecasting and identifying risks, averting risks, transferring risks, spreading risks and controlling risks [7], closely associated with intelligence work, which is all about organizing the intelligence process and utilizing information and data sources, and not just about finding solutions to a certain risk in the short-term [8]. As a matter of fact, by reducing an SEM's level of vulnerability to a risk, its ability to withstand attacks from competitors and the competitive environment will be increased, and its level of resilience will move from average to above average, and the detected threat will be downgraded to the "not immediately at risk".

And for this reason that incomplete information and asymmetrical information probably result in risks, SMEs that have access to risk information often identify potential risks more easily and seize more market opportunities than others, which do not have corresponding information. Consequently, the fact is that the above mentioned model of risk prevention could be carried out successfully based on having adequate and reliable information in limited response time. Additionally, the principle of competitive intelligence is that monitoring competitive activities should focus on a variety of information needs in the different section of risk prevention. The concrete steps of preventing risks of SMEs are as follows:

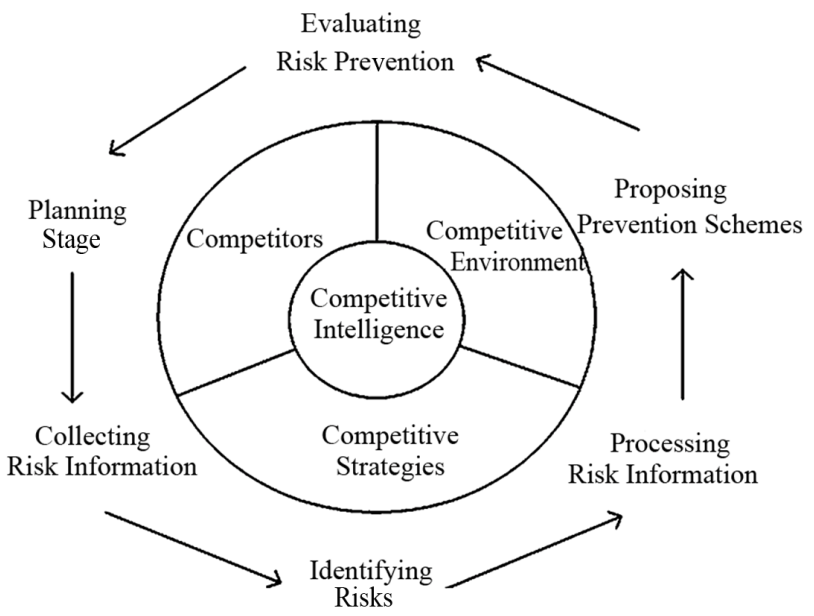

Figure 1. The model of competitive intelligence monitoring in risk prevention of SMEs

\subsection{Planning Stage}

The first step of risk prevention is to plan all activities of risk prevention from the perspective of competitive intelligence-establishing the goal of risk prevention, limiting the scope of risk prevention, defining criteria of risk prevention, making information-gathering plan, making tables of responsibilities and arrangements and so on [9]. According to a study on SMEs of Industry Canada, it is difficult for SMEs not to use competitive intelligence tools but to assure the following issues before: understanding what competitive intelligence is, knowing your own purpose, building internal co-operation, starting with a plan, knowing what you know, protecting your confidentiality, gathering information systematically, analyzing information, building a competitive strategy, understanding the costs and benefits.

In addition, a proactive competitive intelligence operation requires that competitive intelligence professionals, under the direction of a senior manager, take responsibility for coordinating matters relating to intelligence gathering, analysis, interpretation, dissemination, and most importantly, the development of scenarios and future worlds. Furthermore, by embedding the intelligence activities within a well defined ethics policy, competitive intelligence professionals can protect the company's reputation and the individual reputations of those that undertake competitive intelligence work.

To be sure, above activities prepared for risk prevention from the perspective of competitive intelligence, offer right direction or latter specific operation.

\subsection{Collecting Risk Information}

As the basis and foundation of risk prevention activities, collecting risk information is to resolve the problem how 
to collect useful and effective risk information as soon as possible at the minimum cost. Hence, the tasks in this step mainly include the following steps in this stage: analyzing the target of collection, making strategies and plans, confirming collectors and choosing the initial sources or renewable resources of risk information by competitive intelligence-gathering methods.

As we all know, the sources of risk information distribute widely and are viewed as all-embracing, flexible and adaptable. Thus Porter's five-force model is usually regarded as a criterion to establish the sources of risk information [10]. Given that the frequency of risks has the law of distribution- “2-8 principles", collectors should pay more attention to the critical assets of enterprises and discern the three-dimensional values of information, namely, the time, the space and the form. Specifically, it is necessary to search open publications, laws and regulations of government and business as well as many materials of Internet. What is more, some significant information like market competition, strategic decision-making, product structure, investment, liquid assets, human resources, policies and regulations, supply chain management and natural risks, could be extracted by communicating with customers, suppliers, partners, employees of them and competitors, industry associations, experts and organizations.

On the whole, the work of collecting is a dynamic, normative and sequential process. It means that people must make full use of specific methods and tools of competitive intelligence apart from general approaches, such as the traditional method of literature search, network search, telephone interview, direct interview and so on [11]. Besides, the target of collecting should be adjusted in pace with results of analysis and changes in circumstances. Hence collectors should try their best to take full advantage of information technologies so as to optimize channels and methods of collecting.

\subsection{Identifying Risks}

Although the information collected above has certain relevance, it is affected greatly by some subjective factors of the staff. Hence, it is necessary for professionals to recognize threats and vulnerabilities of the enterprise. In other words, keeping close watch on processes and sections of business management, people may identify uncertainties - the sources of risks, and then they can more accurately analyze serious consequences of the risk and more easily find out response measures using various comprehensive methods systematically and continuously.

Risk identification requires professionals consider the characteristics of SMEs, that is, they need to analyze and investigate the personnel, assets and business activities of SMEs comprehensively, mainly concentrating on the following problems: what are current and potential risks? Which one deserves to be researched? What is the main reason for the risk of accidents? What are consequences of the risk? Are all of the measures of identifying risk management proper? [12]

On the one hand, professionals need perceptual knowledge and experience to identify risk information; and on the other hand, more importantly, SMEs must analyze, summarize and sort out the objective statistical data, business information and other risk records to recognize the damage and regularity of the risk. This requires SMEs use some excellent methods of competitive intelligence, for example, analysis of critical success factors, analysis of strategic groups, value chain analysis, SWOT, reverse engineering etc. In addition, other proper methods, including environmental analysis, analysis of financial statements, flow chart of law, decision analysis, dynamic analysis, literature survey and expert survey methods and so on [13], are important tools of analysis. However, each method is not an isolated. A variety of methods are used together simultaneously based on the specific circumstances, which helps to identify risks from the multi-angle and multi-level perspective on the premise of performing relevant work in a comprehensive, institutional and combined way.

\subsection{Processing Risk Information}

By this time, there is sporadic and disorderly risk information, which still cannot be used as the basis of managers' decision-making. Instead, SMEs must assort data systematically and analyze the identified risk information deeply once again. That is to say, choose and classify risk information according to the three standards - the predictability of risk, the probability of occurrence and the extent of loss. Through these activities of classification, sorting and comparison, all risk information is made to a table, which includes the following contents: the name of risk, the description of characteristics, the level of risk, the probability, the cause and the impact, and the degree of the impact.

The above processes mainly depend on some modern information processing technologies. Firstly, it is appropriate to sort out the information collected before by the methods of automatic indexing, automatic classification, data storage technology and computer technology of sorting. Furthermore, the technologies of data mining, online analytical processing, information fusion and case-based reasoning are favorable to extract useful intelligence from a large number of risk information based 
on analyzing the risk information from multi-angle and multi-directional perspective. Learning from the past experience, relevant personnel could uncover some new regulations and knowledge, which would eventually be transformed into high-value competitive intelligence. However, professional experience is vital to manage risk information. It not only helps to control SMEs' different risks appropriately in line with the strategy of hierarchy, but also emphasizes these critical factors of risk information management resulting in high efficiency of risk prevention.

\subsection{Proposing Risk Prevention Schemes}

Based on above steps, decision-makers may put out the corresponding schemes of risk prevention relying on their knowledge and experience. It requires prevention schemes clearly specify the objects, measures, contents and strategies of prevention, all of which are closely related to competitive intelligence. And these schemes do not emphasize efficiency of the forecast, identification, evasion, transfer, spread and the control of risk effectively, but also focus on economic efficiency, that is, the proper scheme is beneficial to obtain the effectiveness of risk prevention at the lowest cost.

\subsection{Evaluating Prevention Schemes}

In fact, SMEs may face potential or new risks along with time. Thus they need to be sensitive to some subtle changes. Once the scheme deviated from the original target, it would improve the scheme in time to ensure the effectiveness of risk prevention.

Therefore, evaluators ought to take full advantage of competitive intelligence as an effective tool in the process of evaluation. Monitoring environment and tracking competitors continuously, competitive intelligence could readily discern some critical factors of environment changes that are enterprise-related internally and externally, so as to identify early-warning signals of potential risks and additional risks. Through the analysis and processing of competitive intelligence, evaluators could determine whether SMEs need to adjust or completely change the primary scheme and they would more easily find out the main reason why the results deviated from the target. It can be said that the whole activities need to consider the problem whether the scheme is best cost-effective and another one is whether the scheme is consistent with the overall strategy of the SMEs.

\section{Conclusions}

In a word, SMEs have smaller scale, inferior economic strength, more difficult of access to information and more imperfect competitive intelligence systems, all of which have resulted in weaker ability to resist risks. Starting from a good many characteristics of SMEs, this paper firstly pointed out how many kinds of risks the SMEs are facing, and then analyzed the demands for competitive intelligence in the risk prevention of SMEs. After that, it was proposed that competitive intelligence plays an important role in the risk prevention of SMEs. By studying the mechanism and procession of competitive intelligence prevention comparatively with larger enterprises, the model that includes the contents of competitive intelligence monitoring in the risk prevention of SMEs were put forward at last. The model may bring into play their strengths and while avoid disadvantages in the increasingly fierce competition. Clearly, it could promote the competitive intelligence system of SMEs to enhance their abilities of risk prevention and ultimately to improve SMEs' competitiveness.

\section{Acknowledgements}

This paper is supported by the project of Program for New Century Excellent Talents in University under grant NCET-06-0624, the key project of National Natural Science Foundation of China (NSFC) under Grant 70833005, and the MOE Project of Key Research Institute of Humanities and Social Sciences at Universities under grant 06JJD870007.

\section{REFERENCES}

[1] X. J. Zha, “Information analysis and forecasting,” Wuhan University Press, Wuhan, 2000.

[2] "Small and medium-sized enterprises," http://baike.baidu.com/view/58855.htm.

[3] “Competitiveness of SMEs in China rose," http://www.cass.net.cn/file/20090625235940.html.

[4] S. Ghoshal, "Global strategy: An organizing framework," Blackwell, Oxford, 2004.

[5] R. A. Jones, N. L. Jimmieson, and A. Griffiths, "The impact of organizational culture and reshaping capabilities on change implementation success: The mediating role of readiness for change,” Journal of Management Studies, Vol. 42, No. 2, pp. 361-86, 2005.

[6] Y. -I. Lee and P. R. J. Trim, "Retail marketing strategy: The role of marketing intelligence, relationship marketing and trust,” Marketing Intelligence \& Planning, Vol. 24, No. 7, pp. 730-745, 2006.

[7] "The measures of preventing the risks of small and medium-sized enterprises in United States," http://www.ceol-awyer.com/news_view.asp?newsid=1178. 
[8] L. M. Fuld, “The new competitor intelligence,” Wiley, Chichester, 1995.

[9] “Competitive intelligence,"

http://www.ic.gc.ca/epic/site/dir-ect.nsf/vwapj/ci-vc_eng. pdf/\$FILE/ci-vc_eng.pdf.

[10] Z. J. Wang, “Competitive intelligence,” Science and Technology Literature Press, Beijing, 2005.

[11] X. B. Jia, "Research on the theory and practice of com- petitive intelligence,” Xi'an Jiaotong University Press, Xi'an, 2006.

[12] X. G. Li, "Industrial development and risk management," China Modern Economic Publishing House, Beijing, 2002.

[13] T. Wu, "The methods and strategies of technological innovation in risk management," Science of Science and Management of S.\&.T., Vol. 5, pp. 45-47, 2006. 